\title{
ENGEVISTA
}

Página da revista: http://www.uff.br/engevista/seer/

\section{Avaliação de solventes e condições experimentais para extração do óleo de sementes de nabo forrageiro (Raphanus sativus $\mathbf{L}$.)}

\author{
Camila da Silva ${ }^{1}$ \\ Sandra Beserra Silva ${ }^{2}$
}

\begin{abstract}
Resumo: Este trabalho teve como objetivo avaliar a eficiência de diferentes solventes e condições experimentais para a extração do óleo de sementes de nabo forrageiro (Raphanus sativus L.). Os experimentos foram realizados a baixa pressão em agitador orbital, avaliando os solventes: etanol, acetato de etila, metanol, metil acetato e $n$-hexano. Os resultados reportam maiores rendimentos em óleo na utilização dos solventes: acetato de etila, $n$-hexano e metil acetato $(\mathrm{p}<0,05)$. Para o solvente selecionado, acetato de etila, avaliou-se o efeito da granulometria das sementes e das variáveis operacionais (temperatura, tempo e volume de solvente) no rendimento em óleo, bem como comparação dos resultados obtidos com a extração convencional utilizando $n$-hexano como solvente. Os resultados obtidos relatam maiores rendimentos utilizando partículas com diâmetro médio de $0,39 \mathrm{~mm}$ e as variáveis operacionais avaliadas apresentaram efeito positivo e significativo, obtendo rendimento de $\sim 32 \%$ em 4 horas de extração, temperatura de $60{ }^{\circ} \mathrm{C}$ e razão solvente/semente de $2 \mathrm{~mL}$ $\mathrm{g}^{-1}$, rendimento semelhante ao obtido pelo método de Soxhlet ( 34\%), em menor tempo de extração e baixo volume de solvente. Os resultados da composição em ácidos graxos dos óleos provenientes das diferentes técnicas de extração não apresentaram diferenças significativas, sendo que em ambos os casos houve predominância dos ácidos graxos monoinsaturados (67\%) e de ácidos graxos poli-insaturados ( 25\%).
\end{abstract}

Palavras-chave: Acetato de etila, extração, nabo forrageiro.

\footnotetext{
${ }^{1}$ UEM - Universidade Estadual de Maringá
}

${ }^{2}$ UEM - Universidade Estadual de Maringá

ENGEVISTA, V. 18, n. 2, p. 348-362, Dezembro 2016. 
ISSN: $1415-7314$

ISSN online: 2317-6717

Abstract: This work aimed to evaluate the efficiency of different solvents and experimental conditions for the extraction of oil from radish seed (Raphanus sativus L.). The experiments were performed at low pressure in an orbital shaker, evaluating solvents: ethanol, ethyl acetate, methanol, methyl acetate and $n$-hexane. The results report higher oil yields in the use of the solvents ethyl acetate, n-hexane and methyl acetate as $(\mathrm{p}<0.05)$. For the selected solvent, ethyl acetate, evaluated the effect of particle size of the seed and the operating variables (temperature, time and solvent volume) in oil yield, as well as comparing the results obtained with the conventional extraction using $n$-hexane as solvent. The results reported higher yield using seed classified in particle with diameter of $0.39 \mathrm{~mm}$ and operating variables evaluated showed positive and significant effect, achieving $\sim 32 \%$ of yield in 4 hours of extraction at $60{ }^{\circ} \mathrm{C}$ and the ratio solvent/seed $2 \mathrm{~mL} \mathrm{~g}^{-1}$, similar yield to that obtained by the Soxhlet method $(\sim 34 \%)$, in less time and low volume of solvent. The results of the fatty acid composition of oil from different extraction techniques showed no significant differences, and in both cases, there was a predominance of monounsaturated fatty acids $(67 \%)$ and polyunsaturated fatty acids $(\sim$ $25 \%)$.

Keywords: Ethyl acetate, extraction, Raphanus sativus L. 


\section{Introdução}

A maior parte de toda a energia consumida no mundo provém de fontes de combustíveis fósseis, maiores responsáveis pelas emissões de gases causadores do efeito estufa. Essas fontes são limitadas com previsão de esgotamento no futuro, portanto, a busca por fontes alternativas de energia é de suma importância (Avhad e Marchetti 2015). Considerada uma energia alternativa, o biodiesel poderá ser uma resposta à problemática ambiental do uso dos combustíveis fósseis (Liaquat et al., 2010).

Atualmente, em torno de $95 \%$ do biodiesel produzido mundialmente é obtido a partir de óleos comestíveis (Abdelmoez et al., 2015), sendo incentivada a busca por novas oleaginosas que produzam óleos não destinados para o consumo humano e que permitam a obtenção do biodiesel dentro das normas internacionais de qualidade. Culturas pouco conhecidas no Brasil, como o naboforrageiro (Raphanus sativus L.), despontam como alternativa interessante para a produção de biodiesel (Wang et al., 2000; Roscoe et al., 2007) e as sementes apresentam de 35\% a $40 \%$ do seu peso em óleo (Domingos et al., 2008; Souza et al., 2009).

O nabo forrageiro é uma planta perene da família Brassicaceae (Domingos et al., 2008) que apresenta tolerância considerável para baixas temperaturas, excelente capacidade para reciclar micronutrientes de solo, apresenta rápido crescimento (150-200 dias para o ciclo completo), baixa exigências agronômicas e um baixo custo de cultivo por hectare. Este cultivar tem sido amplamente utilizado nas regiões Sul e Centro-Oeste do Brasil, como cultura de cobertura em épocas de entressafra e como fertilizante natural verde, pois suas raízes descompactam o solo, permitindo um preparo biológico do mesmo na rotação de culturas e na alimentação animal.

Entre os métodos para extração de óleo de sementes oleaginosas podemos citar a extração por prensagem e extração com solventes como técnicas convencionais, porém a extração com solvente orgânico tem como principais restrições a possível contaminação ambiental, extração de compostos indesejáveis, aumentando o custo de purificação e diminuindo o rendimento dos compostos de interesse, a presença no extrato de resíduos do solvente empregado, o que reduz a qualidade do produto devido à toxicidade do solvente (Mezzomo et al., 2012).

Buscando melhorar a eficiência do processo de extração e a obtenção de rendimentos satisfatórios em condições operacionais amenas, tecnologias menos agressivas ao meio ambiente tem impulsionado estudos envolvendo métodos alternativos, utilizando como solventes substâncias inofensivas ao meio-ambiente e à saúde humana (Tian et al., 2013). A extração do óleo de sementes oleaginosas a baixa pressão em equipamento de agitação orbital é uma alternativa aos métodos tradicionais. Buchs et al. (2007) reportam que este método é de fácil manuseio, rápido, eficiente, apresenta possibilidade da realização de experimentos paralelos e baixo custo operacional. A agitação favorece a circulação convectiva na maior parte do solvente e, assim, compensa a diminuição gradual causada pelo aumento da concentração do soluto (Franco et al., 2007). A extração em agitador orbital utilizando solventes é uma técnica comumente utilizada, que

ENGEVISTA, V. 18, n. 2, p. 348-362, Dezembro 2016. 
permite remover praticamente todo o óleo das sementes, sendo interessante que o solvente utilizado no processo seja pouco dispendioso e não tóxico (Ramluckan et al., 2014).

O solvente acetato de etila tem recebido, ao longo da última década, grande atenção para produção de biodiesel, aliado a extração reativa, pois atua simultaneamente como extrator e reagente, obtendo como coproduto a triacetina que pode ficar presente no biodiesel, atuando como aditivo com elevado valor comercial (Du et al., 2004).

Diante do exposto, este trabalho priorizou como objetivo a extração do óleo das sementes do nabo forrageiro a baixa pressão em equipamento de agitação orbital, bem como a comparação dos rendimentos com a extração convencional utilizando $n$-hexano. Em um primeiro momento, verificou-se a eficiência de diferentes solventes para extração do óleo e para o solvente selecionado realizou-se ensaios para constatar o efeito do diâmetro médio das sementes e variáveis operacionais (temperatura, tempo e volume de solvente) no rendimento em óleo. Os extratos obtidos na condição otimizada no agitador orbital e pelo método convencional foram caracterizados em relação à composição de ácidos graxos e propriedades físico-químicas.

\section{Materiais e métodos}

Foram utilizadas sementes de nabo-forrageiro (Raphanus sativus L.), cultivar IPR 116 (adquirido do fornecedor BRSEEDS SP), as quais apresentaram composição de: 6,66\% $\pm 0,15$ de umidade, 4,12\% $\pm 0,07$ de cinzas, $29,12 \% \pm 0,07$ de proteína e 4,31\% $\pm 0,07$ de fibra bruta, determinada de acordo com IAL (2008). Para as extrações foram utilizados os solventes: acetato de etila (Anidrol), $n$-hexano (Anidrol), metanol (Vetec), etanol (JT Backer) e metil acetato (Sigma Aldrich). Todos os solventes e reagentes utilizados neste estudo foram de grau analítico. As sementes foram trituradas em moinho elétrico (Marconi) e classificadas usando peneiras da série Tyler (Bertel, ASTM).

A extração do óleo das sementes de nabo forrageiro foi realizada em agitador orbital (Marconi, MA 839/A) com controle de temperatura e agitação, onde $4 \mathrm{~g}$ de sementes foram colocadas em Erlenmeyers com tampa de vidro $(250 \mathrm{~mL})$ juntamente com os solventes, utilizando agitação de $100 \mathrm{rpm}$ para todos os experimentos. Após o período de extração as amostras foram filtradas e o excesso de solvente, no filtrado, foi evaporado em rota evaporador (Marconi, MA120) e o remanescente mantido em estufa até peso constante $\mathrm{O}$ cálculo do rendimento em óleo foi realizado conforme Equação 1:

$$
\text { Rendimento em óleo }(\mathrm{Y})=\frac{\mathrm{m}_{2}}{\mathrm{~m}_{1}} \times 100
$$

onde: $\mathrm{m}_{2}$ e $\mathrm{m}_{1}$ são as massas de óleo extraídas e a massa de sementes utilizada nos experimentos, respectivamente.

Primeiramente, avaliou-se a eficiência de diferentes solventes e granulometria na extração do óleo, mantendo fixa as variáveis temperatura $\left(40{ }^{\circ} \mathrm{C}\right)$, razão solvente/semente $\left(4 \mathrm{~mL} \mathrm{~g}^{-1}\right) \mathrm{e}$ 
tempo (4 horas).

Um planejamento experimental Box-Behnken foi empregado para determinar a influência dos fatores principais, tempo, temperatura e razão solvente/semente, no rendimento em óleo para o solvente selecionado. Os níveis testados das variáveis independentes são apresentados na Tabela 1. O software Statistica 8.0 (STATSOFT TM, Inc) foi utilizado para a análise estatística dos dados, utilizando intervalo de $95 \%$ de confiança.

Tabela 1. Variáveis e níveis analisados no planejamento experimental Box-Behnken.

\begin{tabular}{lccc}
\hline \multirow{1}{*}{\multicolumn{1}{c}{ Variáveis }} & \multicolumn{3}{c}{ Nível } \\
\cline { 2 - 4 } & $\mathbf{- 1}$ & $\mathbf{0}$ & $\mathbf{1}$ \\
\hline Tempo $(\mathrm{h})-\mathrm{X}_{1}$ & 2 & 3 & 4 \\
Temperatura $\left({ }^{\circ} \mathrm{C}\right)-\mathrm{X}_{2}$ & 40 & 50 & 60 \\
$\begin{array}{l}\text { Razão solvente/semente } \\
\left(\mathrm{mL} \mathrm{g}^{-1}\right)-\mathrm{X}_{3}\end{array}$ & 1 & 2 & 3 \\
\hline
\end{tabular}

Paralelamente, determinou-se o teor de óleo nas sementes pelo método convencional por Soxhlet, utilizando $n$-hexano como solvente, de acordo com o método descrito pela AOCS (1990).

Os extratos obtidos na condição otimizada e pelo método Soxhlet foram caracterizados utilizando os métodos oficiais recomendados pela AOCS (1990) em termos de índice de acidez (AOCS Ca 5a-40), índice de refração (AOCS Cc 7-25), índice de saponificação (AOCS Cd 3c-91) e índice de peróxidos (AOCS Cd 8-53), índice de iodo foi determinado pelo Método de Wijs conforme (Cd 1-25) e o teor de água foi determinado em um titulador Karl Fischer (Orion, AF8). A composição em ácidos graxos dos óleos foram determinadas por cromatografia gasosa utilizando o método descrito por Garcia et al. (2012).

\section{Resultados e discussão}

\subsection{Seleção do Solvente}

Os resultados de rendimento em óleo obtidos utilizando diferentes solventes e seus respectivos índices de polaridade e viscosidade estão apresentados na Tabela 2. Os resultados obtidos indicam maiores rendimentos para a utilização do acetato de etila, $n$-hexano e metil acetato, não sendo observada diferença significativa entre estes solventes. Desta forma, verifica-se que solventes apolares e com baixa polaridade apresentam maior eficiência na extração do óleo de nabo forrageiro, visto que o rendimento da extração depende das diferentes interações entre soluto e solvente (Almeida et al., 2012), do tipo de lipídeos presentes, bem como a proporção de lipídeos polares e não polares presentes na matéria-prima. A viscosidade do solvente também está entre as propriedades que afetam a extração, visto que solventes com baixa viscosidade apresentam alta difusividade, facilitando a difusão dentro dos poros da matriz vegetal (Rezaie et al., 2015). 
Tabela 2. Resultados de rendimento em óleo utilizando diferentes solventes.

\begin{tabular}{lccc}
\hline \multicolumn{1}{c}{ Solvente } & $\begin{array}{c}\text { Viscosidade } \\
(\mathbf{m P a . s})\end{array}$ & Polaridade & $\begin{array}{c}\text { Rendimento } \\
(\boldsymbol{\%})^{*}\end{array}$ \\
\hline$n$-hexano & $0,32^{2}$ & 0 & $16,89 \pm 0,81^{\mathrm{a}}$ \\
Metil acetato & $0,37^{1}$ & 3,5 & $17,04 \pm 0,27^{\mathrm{a}}$ \\
Acetato de etila & $0,43^{1}$ & 4,4 & $16,39 \pm 0,45^{\mathrm{a}}$ \\
Metanol & $0,51^{1}$ & 5,1 & $7,62 \pm 0,07^{\mathrm{b}}$ \\
Etanol & $1,08^{1,3}$ & 5,2 & $9,54 \pm 0,13^{\mathrm{c}}$ \\
\hline
\end{tabular}

${ }^{1}$ Gonzáles et al. (2007); ${ }^{2}$ Dubey e Sharma (2008); ${ }^{3}$ Baltazar et al. (2013).

* Médias seguidas pela mesma letra não se diferem estatisticamente $(\mathrm{p}>0,05)$.

Estudos similares foram realizados por Rezaie et al. (2015) que avaliaram a eficiência de diferentes solventes na extração de óleo de pistácia, reportando elevados rendimentos ( 36\%) na extração com acetato de etila. Tian et al. (2013) avaliaram a eficiência de diferentes solventes na extração de óleo de sementes de romã e obtiveram $20,04 \%$ e 20,48\% de rendimento em óleo com acetato de etila e n-hexano, respectivamente, relatando que as diferenças na eficiência dos solventes na extração de óleo estão relacionadas com a polaridade e a viscosidade do solvente.

Considerando que não foi observada diferença significativa entre os resultados com acetato de etila, metil acetato e $n$-hexano ( $>0,05)$, o acetato de etila torna-se viável por apresentar características eficientes, solubilidade em óleo e seletividade, estáveis propriedades físicoquímicas, baixo ponto de ebulição, recuperação fácil, não toxidade e principalmente ser de baixo custo (Zhang e Zhao, 2006). O acetato de etila é reportado na literatura como solvente de destaque, atuando simultaneamente como extrator e reagente (Modi et al., 2007), sendo que o $n$-hexano é apenas utilizado como solvente para extração do óleo.

\subsection{Efeito da Granulometria}

Na Tabela 3 são apresentados os dados de rendimento em óleo obtidos com diferentes granulometrias utilizando acetato de etila como solvente. Pode ser verificado a partir dos resultados que as extrações realizadas com as sementes classificadas com diâmetro médio de 0,39 mm apresentaram maior rendimento em óleo. Tal fato pode ser explicado, pois com a diminuição do diâmetro das sementes aumenta-se a superfície de contato entre o solvente e as sementes, aumentando assim a taxa de extração (Franco et al., 2007).

Tabela 3. Rendimentos em óleo utilizando diferentes granulometrias.

\begin{tabular}{cc}
\hline $\mathbf{D}(\mathbf{m m})^{*}$ & ${\text { Rendimento }(\%)^{* *}}^{* *}$ \\
\hline 0,71 & $16,44 \pm 0,04^{\mathrm{a}}$ \\
0,54 & $25,47 \pm 0,80^{\mathrm{b}}$ \\
0,39 & $29,72 \pm 0,45^{\mathrm{c}}$
\end{tabular}

"Diâmetro médio;** Médias seguidas pela mesma letra não se diferem estatisticamente $(\mathrm{p}>0,05)$.

Eikani et al. (2011) avaliaram o efeito da granulometria na extração do óleo de sementes de romã, obtendo $22 \%$ de óleo com partículas de menor diâmetro, 0,25 mm, enquanto que as 
partículas com diâmetro de 1,0 mm apresentaram 11,26\% de rendimento. Nagy et al.(2008) investigaram o efeito da granulometria de sementes de páprica (Capsicumannuum L.) no rendimento da extração, reportando $90 \%$ e $45 \%$ de óleo com partículas de 0,2 mm e 1,5 mm, respectivamente.

\subsection{Efeitos das Variáveis Operacionais}

A Tabela 4 apresenta as condições experimentais para o planejamento Box-Behnken, os resultados de rendimento em óleo utilizando sementes com diâmetro médio de 0,39 $\mathrm{mm}$ e acetato de etila como solvente, bem como o rendimento obtido utilizando o método por Soxhelt, para efeito comparativo. O maior rendimento, 32,61\%, foi obtido a $60{ }^{\circ} \mathrm{C}$, 4 horas e razão solvente/semente de $2 \mathrm{~mL}$ g-1, demonstrando que a técnica de extração em agitador orbital é viável, obtendo 93,3\% de rendimento do método convencional, ressaltando que tal técnica utiliza menores tempos de extração e menor volume de solvente.

Tabela 4. Condições experimentais e rendimento em óleo na avaliação do efeito das variáveis operacionais.

\begin{tabular}{ccccc}
\hline \multirow{2}{*}{ Experimento } & \multicolumn{3}{c}{ Variáveis } & \multirow{2}{*}{$\mathbf{Y}(\boldsymbol{\%})$} \\
\cline { 2 - 4 } & $\mathbf{X}_{\mathbf{1}}$ & $\mathbf{X}_{\mathbf{2}}$ & $\mathbf{X}_{\mathbf{3}}$ & \\
\hline $\mathbf{1}$ & -1 & -1 & 0 & 25,99 \\
$\mathbf{2}$ & 1 & -1 & 0 & 28,26 \\
$\mathbf{3}$ & -1 & 1 & 0 & 30,99 \\
$\mathbf{4}$ & 1 & 1 & 0 & 32,61 \\
$\mathbf{5}$ & -1 & 0 & -1 & 23,34 \\
$\mathbf{6}$ & 1 & 0 & -1 & 23,86 \\
$\mathbf{7}$ & -1 & 0 & 1 & 29,30 \\
$\mathbf{8}$ & 1 & 0 & 1 & 30,27 \\
$\mathbf{9}$ & 0 & -1 & -1 & 23,62 \\
$\mathbf{1 0}$ & 0 & 1 & -1 & 23,80 \\
$\mathbf{1 1}$ & 0 & -1 & 1 & 28,58 \\
$\mathbf{1 2}$ & 0 & 1 & 1 & 31,08 \\
$\mathbf{1 3}$ & 0 & 0 & 0 & $31,58 \pm 0,25^{1}$ \\
\hline \multicolumn{5}{c}{ Soxhlet } \\
\end{tabular}

${ }^{1}$ média de quatro experimentos.

${ }^{2}$ Obtido com $n$-hexano como solvente (30:1 razão volume de solvente/massa de semente) em refluxo por 8 hora.

Oliveira et al. (2013) estudaram o efeito das variáveis operacionais na extração de óleo de semente de maracujá em agitador orbital e por Soxhlet. Os autores relatam que o melhor rendimento em agitador orbital, $\sim 18 \%$ em óleo, foi obtido empregando menor quantidade de solvente (1:4) em 4 horas de extração, comparado com a extração por Soxhlet, que apresentou rendimento de $\sim 14 \%$ em 16 horas, utilizando razão solvente:semente (30:1).

A Tabela 5 apresenta o efeito das variáveis independentes sobre o rendimento em óleo. Para as condições experimentais utilizadas no planejamento a razão solvente/semente, o tempo e a

ENGEVISTA, V. 18, n. 2, p. 348-362, Dezembro 2016. 
temperatura apresentaram efeito significativo $(\mathrm{p}<0,05)$ no rendimento em óleo.

Tabela 5. Efeitos das variáveis independentes na extração conduzida em agitador orbital do óleo de nabo forrageiro.

\begin{tabular}{ccccc}
\hline Variável & Efeito & p-valor & Coeficiente & Erro padrão \\
\hline Média/intercepto & 27,64 & $>0,001$ & 27,64 & 0,09 \\
Tempo $-\mathrm{X}_{1}(\mathrm{~L})$ & 1,34 & 0,0101 & 0,67 & 0,23 \\
Tempo $-\mathrm{X}_{1}(\mathrm{Q})$ & 1,10 & 0,0066 & 0,55 & 0,16 \\
Temperatura - $\mathrm{X}_{2}(\mathrm{~L})$ & 3,00 & 0,0009 & 1,50 & 0,23 \\
Temperatura $-\mathrm{X}_{2}(\mathrm{Q})$ & 1,02 & 0,0082 & 0,51 & 0,16 \\
Solvente $-\mathrm{X}_{3}(\mathrm{~L})$ & 6,15 & 0,0001 & 3,07 & 0,23 \\
Solvente $-\mathrm{X}_{3}(\mathrm{Q})$ & 3,79 & 0,0001 & 1,90 & 0,16 \\
$\mathrm{X}_{1} \times \mathrm{X}_{2}$ & $-0,32$ & 0,4001 & $-0,16$ & 0,33 \\
$\mathrm{X}_{1} \times \mathrm{X}_{3}$ & 0,22 & 0,5410 & 0,11 & 0,33 \\
$\mathrm{X}_{2} \times \mathrm{X}_{3}$ & 1,16 & 0,0377 & 0,58 & 0,33 \\
\hline
\end{tabular}

${ }^{a}$ significância estatística $(\mathrm{p}<0,05)$.

O efeito da quantidade de solvente é claramente observado quando comparamos os resultados dos experimentos 5-7 e 9-11. Zhang et al. (2009) relatam que o aumento da quantidade de solvente pode melhorar a difusão através da redução da viscosidade, promover maior concentração do solvente no interior da matriz vegetal e resultar no aumento da eficiência da extração. Goula (2013) avaliou o efeito da quantidade de solvente empregado na extração do óleo de sementes de romã e observou que o acréscimo de solvente favoreceu o aumento no rendimento em óleo utilizando razão solvente:semente de $20: 1$, a $50{ }^{\circ} \mathrm{C}$ e 40 minutos de extração assistida por ultrassom. O efeito da temperatura pode ser atribuído ao aumento da solubilidade do óleo com o acréscimo da temperatura, visto que a viscosidade e a densidade do solvente diminuem, resultando no aumento das taxas de transferência de massa (Hemwimol et al., 2006). Samaram et al. (2015) citam que a extração conduzida em temperaturas elevadas proporciona a ruptura dos tecidos vegetais e melhora a difusão, promovendo a eluição do óleo ligado no interior da semente para o meio. O efeito da temperatura é verificado ao observarmos os rendimentos obtidos nos experimentos 2-4 e 11-12.

Suganya e Renganathan (2012) avaliaram a influência da temperatura na extração do óleo de algas e reportaram, que utilizando razão solvente: biomassa de 5:1 e 120 minutos de extração, $\sim 7 \%$ e $9,5 \%$ de rendimento em óleo quando empregadas as temperaturas de $35{ }^{\circ} \mathrm{C}$ e $55^{\circ} \mathrm{C}$, respectivamente.

Para o rendimento em óleo pode-se observar que a interação entre temperatura e razão solvente/semente influenciou positivamente e significativamente na resposta $(\mathrm{p}<0,05)$. Segundo Stanisavljević et al. (2007) ao aumentar a quantidade de solvente aumenta-se a solubilidade em óleo, devido o aumento da força motriz que promove a transferência de massa em elevadas temperaturas, porém, ao atingir o equilíbrio é inviável a utilização de quantidades excessivas de solvente. 
ISSN: $1415-7314$

ISSN online: $2317-6717$

O efeito do tempo de extração é observado, por exemplo, na comparação dos experimentos 3-4 e 1-2. Li et al. (2004) ao avaliarem a influência do tempo empregado na extração do óleo de grãos de soja, utilizando uma mistura dos solventes $n$-hexano e isopropanol, concluíram que conforme aumenta-se o tempo da extração, de 120 para 180 minutos, ocorre o aumento significativo no rendimento, de $33 \%$ para $\sim 39 \%$, sob temperatura ambiente $\left(25^{\circ} \mathrm{C}\right)$ auxiliado por sonda ultrassônica e utilizando razão solvente/grão de $1,5 \mathrm{~mL} \mathrm{~g}^{-1}$.

O efeito do tempo de extração pode ser evidenciado para utilização de outros solventes. Mani et al. (2007) utilizando $n$-hexano, na extração do óleo de sementes de moringa (Moringa Oleifera), relatam que o aumento do tempo de 120 minutos para 240 minutos favoreceu o rendimento em óleo, obtendo-se $\sim 33,1 \%$ em $60^{\circ} \mathrm{C}$, razão solvente/semente $12,5 \mathrm{~mL} \mathrm{~g}^{-1}$.

Com base nos coeficientes de regressão, com significância estatística, apresentados na Tabela 5 foi obtido o modelo matemático empírico (Equação 2), que correlaciona o rendimento em óleo, variável resposta, com as variáveis independentes.

Posteriormente ao estudo dos coeficientes de regressão, realizou-se a análise de variância (ANOVA) com aplicação do teste $\mathrm{F}$, indicando uma boa concordância, sendo que análise da estatística "F" apresentou valor de F calculado $(28,02)$ maior que o $F$ tabelado $(3,50)$. Além disso, a regressão linear explica $96,17 \%$ das variações entre os valores experimentais e os previstos pelo mesmo a um nível de significância de $(95 \%)$, validando o modelo matemático apresentado na Equação 2.

$$
Y(\%)=27,64+0,67 X_{1}+1,50 X_{2}+3,07 X_{3}+0,55 X_{1}^{2}+0,51 X_{2}^{2}+1,90 X_{3}^{2}+0,58 X_{3} X_{2}
$$

Diante do contexto a otimização das variáveis experimentais foi realizada a fim de analisar os valores adequados para cada variável. A Figura 1 demonstra os valores ótimos das variáveis do processo, sendo possível constatar que a equação preditiva apresentou um valor para o rendimento em óleo de 32,95\%. Em relação a este rendimento, três experimentos de verificação foram realizados e apresentaram $32,25 \% \pm 0,113$ de rendimento em óleo.

O rendimento máximo em óleo previsto neste trabalho foi 32,95\% sob condições ideais (Figura 1). Valores semelhantes foram obtidos por Mandal et al. (2002) ao avaliarem os teores de lipídeos de sementes de nabo forrageiro, obtendo teores entre 30,7\% e 35,6\% em óleo. Abdalla et al. (2008), Souza et al. (2009) e Constantino et al. (2014) realizaram a extração do óleo de semente de Raphanus Sativus L. e constataram que as sementes contêm 31, 36,85 e 38,08\% do seu peso em óleo, respectivamente. De acordo com os estudos de Kataoka et al. (2011) a semente de nabo forrageiro apresenta alto teor de óleo, 35\%, similar ao obtido neste trabalho. Guerra e Fuchs. (2009) reportam que sementes de nabo forrageiro apresentam de 35\% a 50\% de óleo, sendo que a diferença nos teores pode esta correlacionada com o método de extração aplicado. 


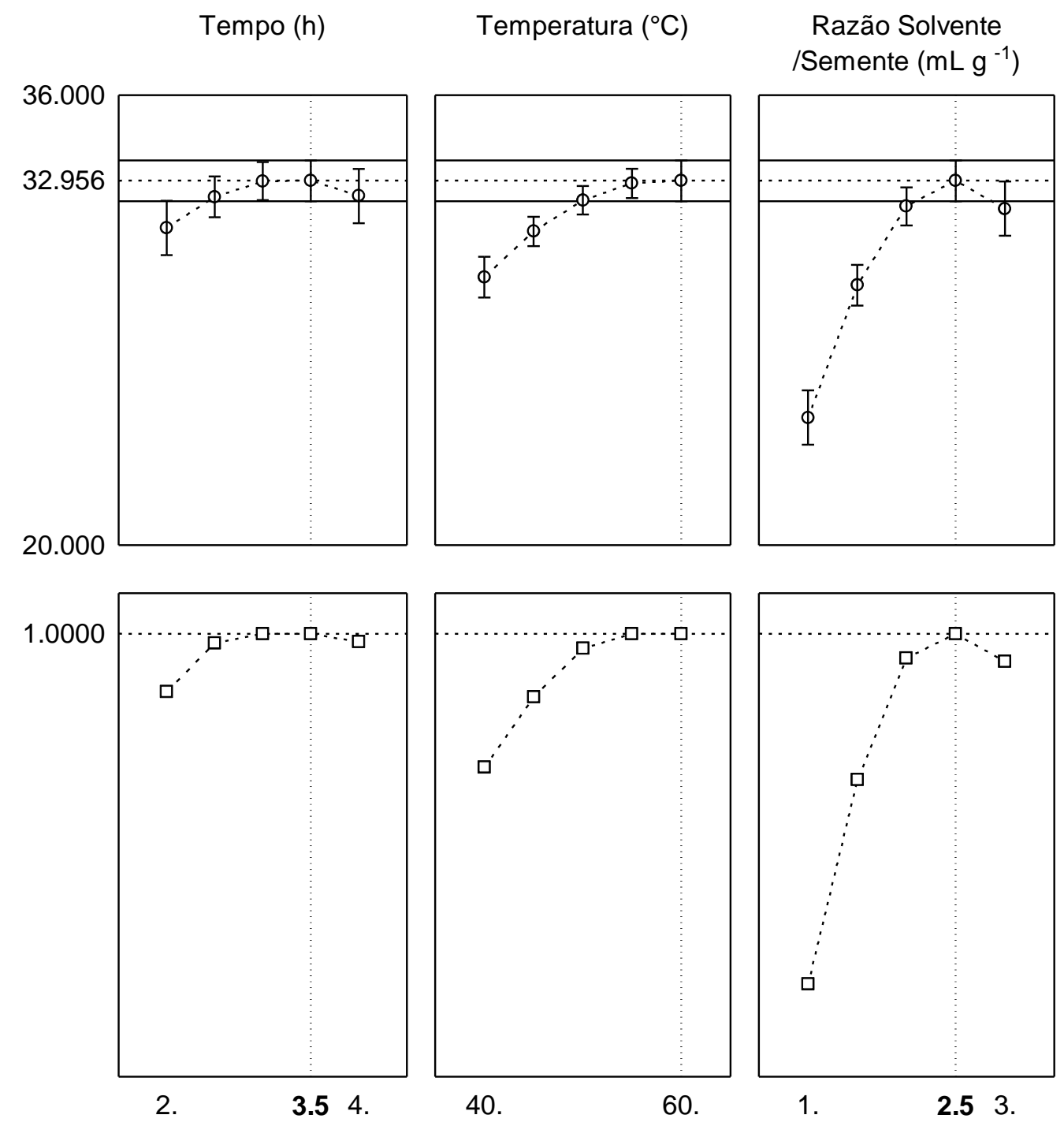

Figura 1. Otimização do rendimento em óleo.

\subsection{Caracterização do Óleo}

A Tabela 6 apresenta o perfil de ácidos graxos dos extratos obtidos pelo método de Soxhlet e na condição otimizada em agitador orbital utilizando os solventes $n$-hexano e acetato de etila, respectivamente, onde se verifica que, em geral, os teores não foram afetados $(p>0,05)$ pela natureza do método de extração. Conforme os resultados apresentados na Tabela 6, os extratos apresentaram elevados teores de ácido oleico $(\sim 34 \%)$, acido erúcico $(\sim 26 \%)$ e ácido linoleico $(\sim 14 \%)$. Pode-se observar que a composição do óleo de nabo forrageiro é predominante por ácidos graxos monoinsaturados, com elevada percentagem do ácido erúcico, sendo este, impróprio para fins alimentícios e despontando como uma fonte promissora para produção de biodiesel (Chammoun et al., 2013). Alguns autores na literatura reportam resultados semelhantes para a composição do óleo de semente de nabo forrageiro. Domingos et al. (2008) relatam que os ácidos graxos oleico $(\sim 35 \%)$, linoleico $(\sim 18 \%)$ e erúcico $(\sim 16,3 \%)$ são os principais encontrados no óleo de Raphanus sativus L. Valle et al. (2010) analisaram a composição do óleo de nabo forrageiro, 
obtendo predominância dos ácidos erúcico (33,3\%), oleico $(27,9 \%)$ e linoléico $(7,6 \%)$. Uluata e Ozdemir (2012) avaliaram a composição dos ácidos graxos do óleo de nabo forrageiro, reportando os principais ácidos linoleico (19,88\%), oleico $(19,08 \%)$ e erúcico $(40,83 \%)$.

Tabela 6. Quantificação de ácidos graxos do óleo de semente de nabo forrageiro obtido a partir da extração com agitador orbital e Soxhlet.

\begin{tabular}{cccc}
\hline Métodos & & Agitador Orbital & Soxhlet \\
\hline & Palmítico & $4,55 \pm 0,19^{\mathrm{a}}$ & $5,44 \pm 0,26^{\mathrm{a}}$ \\
& Linoleico & $14,07 \pm 0,37^{\mathrm{a}}$ & $13,58 \pm 0,73^{\mathrm{a}}$ \\
& Oleico & $33,50 \pm 0,18^{\mathrm{a}}$ & $34,33 \pm 0,73^{\mathrm{a}}$ \\
& & $11,33 \pm 0,57^{\mathrm{a}}$ & $11,21 \pm 0,36^{\mathrm{a}}$ \\
Ácidos & Linolênico & & \\
& & $2,65 \pm 0,12^{\mathrm{a}}$ & $2,58 \pm 0,20^{\mathrm{a}}$ \\
& Esteárico & & \\
& & & \\
& Gondoico & $7,80 \pm 0,17^{\mathrm{a}}$ & $7,17 \pm 0,60^{\mathrm{a}}$ \\
& & & \\
EGS $^{2}$ & & $26,08 \pm 0,48^{\mathrm{a}}$ & $25,67 \pm 2,0^{\mathrm{a}}$ \\
$\mathrm{AGM}^{3}$ & & 7,20 & 8,03 \\
$\mathrm{AGP}^{4}$ & & 67,39 & 67,19 \\
& & 25,41 & 24,79 \\
\hline
\end{tabular}

${ }^{1}$ Resultados em g. $100 \mathrm{~g}^{-1}$ de óleo; ${ }^{2} \mathrm{AGS}$ - ácidos graxos saturados; ${ }^{3}$ AGM-ácidos graxos monoinsaturados; ${ }^{4}$ AGP - ácidos graxos poli-insaturados. Médias seguidas pela mesma letra, em cada linha, não se diferem estatisticamente $(\mathrm{p}>0,05)$.

A caracterização físico-química do óleo é necessária para atestar a sua qualidade, pois é através destes resultados que se estabelece a técnica que deverá ser utilizada para aplicação do mesmo para produção de biodiesel. Os valores obtidos na caracterização físico-química do óleo das sementes de nabo forrageiro, obtido a partir da extração pelo método de Soxhlet e na condição otimizada em agitador orbital utilizando os solventes n-hexano e acetato de etila, respectivamente, são apresentados na Tabela 7.

Tabela 7. Características físico-químicas do óleo das sementes de nabo forrageiro obtido a partir da extração em agitador orbital e Soxhlet.

\begin{tabular}{lcc}
\hline \multicolumn{1}{c}{ Métodos } & Agitador Orbital & Soxhlet \\
\hline Índice de Acidez $\left(\mathrm{mg} \mathrm{de} \mathrm{KOH} \mathrm{g}^{-1)}\right.$ & $2,53 \pm 0,09^{\mathrm{a}}$ & $2,62 \pm 0,03^{\mathrm{a}}$ \\
Índice de Saponificação $\left(\mathrm{mg} \mathrm{de} \mathrm{KOH} \mathrm{g}^{-1}\right)$ & $139,93 \pm 0,13^{\mathrm{a}}$ & $184,74 \pm 0,91^{\mathrm{b}}$ \\
Índice de Iodo $\left(\mathrm{g} \mathrm{de}_{2} 100 \mathrm{~g}^{-1}\right)$ & $100,78 \pm 0,070^{\mathrm{a}}$ & $105,42 \pm 0,08^{\mathrm{b}}$ \\
Índice de Peróxidos $\left(\mathrm{meq} \mathrm{Kg}^{-1}\right)$ & $12,41 \pm 0,009^{\mathrm{a}}$ & $13,83 \pm 0,013^{\mathrm{b}}$ \\
Índice de Refração $\left(\mathrm{a} 25^{\circ} \mathrm{C}\right)$ & $72,30 \pm 0,002^{\mathrm{a}}$ & $72,33 \pm 0,09^{\mathrm{a}}$ \\
Teor de umidade $(\%)$ & $0,234 \pm 0,003^{\mathrm{a}}$ & $0,217 \pm 0,002^{\mathrm{a}}$ \\
\hline
\end{tabular}

*Médias seguidas pela mesma letra, em cada linha, não se diferem estatisticamente $(p>0,05)$. 
Os óleos obtidos a partir dos diferentes métodos apresentaram 0,234 e 0,217\% de umidade, sendo estes valores acima do limite reportado pela literatura para produção de biodiesel, de 0,06\%, o que indica que reações paralelas de saponificação podem ocorrer quando da utilização deste óleo na obtenção deste biocombustível a partir da catálise homogênea básica (Kusdiana e Saka, 2004; Demirbas, 2009). Os óleos obtidos apresentaram índice de peróxido de 12,41 meq $\mathrm{Kg}^{-1}$ e 13,83 meq $\mathrm{Kg}^{-1}$. Os baixos valores encontrados para este óleo são indicativos de boa qualidade já que este parâmetro pressupõe processos degradativos. Ferrari et al. (2005) avaliaram as características físico-químicas do óleo de nabo forrageiro, reportando resultados semelhantes ao deste trabalho, com índice de peróxidos de 12,40 meq $\mathrm{Kg}^{-1}$.

O índice de refração foi de 72,3 para ambos os processos de extração, não apresentando diferença significativa. Este valor também varia de acordo com a qualidade da matéria-prima (Kobori e Jorge, 2005), portanto quanto maior o grau de maturação do fruto do qual o óleo foi extraído, maior o teor de sólidos solúveis.

O índice de iodo do óleo foi de 100,78 e 105, 42 (g de I2 $\left.100 \mathrm{~g}^{-1}\right)$ para a extração conduzida em agitador orbital e Soxhlet, respectivamente. De acordo com Naz et al. (2004) os valores descritos na literatura para o índice de iodo são, em geral, apresentados como uma faixa de valor ao invés de um número fixo, pois o grau de insaturações pode variar de acordo com aspectos ligados a sazonalidade da oleaginosa ou em função do tipo de processamento do óleo.

\section{Conclusão}

A extração de óleo de nabo forrageiro é reportada, sendo que entre os solventes avaliados, o acetato de etila destaca-se com melhor rendimento e por além de solvente poder atuar como reagente. Os resultados relatam que a extração em agitador orbital proporcionou melhor desempenho, quando comparada ao método convencional, em menor tempo e utilizando menor quantidade de solvente. As variáveis operacionais apresentaram efeito positivo e significativo no rendimento, sendo possível obter $\sim 32 \%$ de óleo em 4 horas de extração, temperatura de $60{ }^{\circ} \mathrm{C}$ e razão solvente/semente $2 \mathrm{~mL} \mathrm{~g}^{-1}$. A partir dos resultados verificou-se que a composição em ácidos graxos e características físico-químicas dos óleos obtidos a partir dos diferentes processos não apresentou diferença significativa. 


\section{Referências}

ABDAllA, A. L., FILHO, J. C. S., GODOI, A. R., CARMO, C. A., EDUARDO, J. L. P. 2008. Utilização de subprodutos da indústria de biodiesel na alimentação de ruminantes. Revista Brasileira de Zootecnia, 37, 260-268.

ABDELMOEZ, W., ASHOUR, E., NAGUIB, S. M. 2015. A review on green trend for oil extraction using subcritical water technology and biodiesel production. Journal of Oleo Science, 64, 467-478.

ALMEIDA, P. P., MEZZOMO, N., FERREIRA, S. R. S. 2012. Extraction of Menthaspicata L. volatile compounds: evaluation of process parameters and extract composition. Food and Bioprocess Technology, 5, 548-559.

AOCS - AMERICAN oil chemists society. 1990. Official methods and recommended practices of the American Oil Chemists Society. 4th ed. Champaign, USA.

ANP - Agência Nacional de Petróleo, Gás Natural e Biocombustíveis. Resolução No 42, de 19/04/2005.

AVHAD, M. R., MARCHETTI, J. M. 2015. A review on recent advancement in catalytic materials for biodiesel production. Renewable and Sustainable Energy Reviews, 50, 696-718.

BALTAZAR, A. E., SILVA, G. A. I., CERON, C. C. 2013. Volumetric and Transport Properties of binary mixtures of n-octane + ethanol, +1 - Propanol, +1 - butanol, and $+1-$ pentanol from (293.15 to 323.15) K at atmospheric pressure. Journal of Chemical \& Enginnering Data, 58, 3351 3363.

BUCHS, J., MAIER, U., LOTTER, S., PETER, C. 2007. Calculating liquid distribution in shake flasks on rotary shakers at water like viscosities. Biochemical Engineering Journal, 34, $200-208$. CHAMMOUN, N., GELLER, D. P., DAS, K. C. 2013. Fuel properties, performance testing and economic feasibility of Raphanus sativus (oil seed radish) biodiesel. Industrial Crops and Products, $45,155-159$.

CONSTANTINO, A. F., LACERDA JR, V., SANTOS, R. B., GRECO, S. J., SILVA, R. C., NETO, ÁlVARO, C., BARBOSA, L. L., CASTRO, E. V. R., FREITAS, J. C. C. 2014. Análise do teor e da qualidade dos lipídeos presentes em sementes de oleaginosas por rmn de baixo campo. Química Nova, 37, 10-17.

DEMIRBAS, A. 2009. Biodiesel from waste cooking oil via base-catalytic and supercritical methanol transesterification. Energy Conversion and Management, 50, 923-927.

DOMINGOS, A. K., SAAD, E. B., W, H. M., RAMOS, L. P. 2008. Optimization of the ethanolysis of Raphanus sativus (L.Var.) crude oil applying the response surface methodology. Bioresource Technology, 99, 1837-1845.

DU, W., XU, Y., LIU, D., ZENG, J. 2004. Comparative study on lipase-catalyzed transformation of soybean oil for biodiesel production with different acyl acceptors. Journal of Molecular Catalysis B: Enzymatic, 30, 125-129.

DUBEY, G., SHARMA, M. 2008. Study of molecular interactions in binary liquid mixtures of 1octanol with n-hexane, n-octane, and n-decane using volumetric, viscometric, and acoustic properties. The Journal of Chemical Thermodynamics, 40, $991-1000$.

EIKANI, M. H., GOLMOHAMMAD, F., HOMAMI, S. S. 2011. Extraction of pomegranate (Punicagranatum L.) seed oil using superheated hexane. Food and Bioproducts Processing, 90, 3236.

FERRARI, R. A., ARCE, M. A. B. R., RIBEIRO, F. L. F. Biodiesel de óleo de Raphanus Sativus L. In: II Congresso Brasileiro de Plantas Oleaginosas, Óleos, Gorduras e Biodiesel, 2005, Minas Gerais, Brasil, 743-7748.

FRANCO, D., PINELO, M., SINEIRO, J., NUNEZ, M. J. 2007. Processing of Rosa rubiginosa: Extraction of oil and antioxidant substances. Bioresource Technology, 98, 3506-3512.

GARCIA, V. A. S., CABRAL, V. F., ZANOELO, E., SILVA, C. CARDOZO-FILHO, L. 2012.

Extraction of Mucuna seed oil using supercritical carbon dioxide to increase the concentration of Ldopa in the defatted meal. The Journal of Supercritical Fluids, 69, 75-81.

GONZÁLES, B., CALVAR, N., GÓMEZ, E., DOMINGUEZ, A. 2007. Density, dynamic viscosity, and derived properties of binary mixtures of methanol or ethanol with water, ethyl acetate, and methyl acetate at $\mathrm{T}=(293.15,298.15$, and 303.15) K. The Journal of Chemical Thermodynamics, 39, 1578-1588. 
GOULA, A. M. 2013. Ultrasound-assisted extraction of pomegranate seed oil - Kinetic modeling. Journal of Food Engineering, 117, 492-498.

GUERRA, E. P., FUCHS, W. Mini-usina de óleo vegetal comestível e biocombustível. In: SIMPÓSIO BRASIL-ALEMANHA/ DEUTSCH-BRASILIANISCHES SYMPOSIUM, 4., 2009, Curitiba. Anais. Curitiba: UFPR.

HEMWIMOL, S., PAVASANT, P., SHOTIPRUK, A. 2006. Ultrasound-assisted extraction of anthraquinones from roots of Morinda citrifolia. Ultrasonics Sonochemistry, 13, 543-548.

IAL - INSTITUTO ADOLFO LUTZ. 2008. Métodos físico-químicos para análise de alimentos. São Paulo.

KAOKATA, V. Y., CARVALHO, M. L. M., OLIVEIRA, M. S., CALDEIRA, C. M. 2011. Validação de metodologia para o teste de germinação em sementes de nabo forrageiro (Raphanus sativus L. var. oleiferus). Revista Brasileira de Sementes, 33, 69-79.

KOBORI, C. N., JORGE, N. 2005. Caracterização dos óleos de algumas sementes de frutas como aproveitamento de resíduos industriais. Ciência e Agrotecnologia, 9, 1008-1014.

KUSDIANA, D., SAKA, S. 2004. Effects of water on biodiesel fuel production by supercritical methanol treatment. Bioresource Technology, 91, 289-295.

LI, H., PORDESIMO, L., WEISS, J. 2004. High intensity ultrasound-assisted extraction of oil from soybeans. Food Research International, 37, 731 -738.

LIAQUAT, A. M., KALAM, M. A., MASJUKI, H. H., JAYED, M. H. 2010. Potential emissions reduction in road transport sector using biofuel in developing countries. Atmospheric Environment, 32, 3869-3877.

LIMA, J. R. O., SILVA. R, B., SILVA, C. C. M., SANTOS, L. S., SANTOS Jr. J. R., MOURA, E. M., MOURA, C. V. R. 2007. Biodiesel de babaçu (Orbignya sp.) obtido por via etanólica. Química Nova, 30, $600-603$.

MANDAL, S., YADAV, S., SINGH, R., BEGUM, G., SUNEJA, P., SINGH, M. 2002.

Correlation studies on oil content and fatty acid profile of some Cruciferous species. Genetic

Resources and Crop Evolution, 49, 551-556.

MANI, S., JAYA, S., VADIVAMBAL, R. 2007. Optimization of solvent extraction of moringa (Moringa Oleifera) seed kernel oil using response surface methodology. Food and Bioproducts Processing, 85, 328 -335.

MEZZOMO, N., PAZ, E., MARASCHIN, M., MARTIN, A., COCERO, M. J., FEREEIRA, S. R. S. 2012. Supercritical anti-solvent precipitation of carotenoid fraction from pink shrimp residue: Effect of operational conditions on encapsulation efficiency. The Journal of Supercritical Fluids, 66, 342-349.

MODI, M. K., REDDY, J. R. C., RAO, B. V. S. K., PRASAD, R. B. N. 2007. Lipase-mediated conversion of vegetable oils into biodiesel using ethyl acetate as acyl acceptor. Bioresource Technology, 98, 1260-1264.

NAGAOKA, A. K., SILVEIRA, M. A. 2012. Crescimento, produtividade e plantas daninhas no cultivo de nabo forrageiro em três manejos do solo. Revista Energia na Agricultura, 27, 13-26.

NAGY, B., SIMÁNDI, B. 2008. Effects of particle size distribution, moisture content, and initial oil content on the supercritical fluid extraction of paprika. The journal of Supercritical Fluids, 46, 293-298.

NAZ, S., SHEIKH, H., SIDDIQUI, S. S. A. 2004. Oxidation stability of olive, corn and soybean oil under different conditions. Food Chemistry, 88, 253-259.

OLIVEIRA, J. P., ANTUNES, P. W. P., PINOTTI, L. M., CASSINI, S. T. A. 2014. Caracterização físico-química de resíduos oleosos do saneamento e dos óleos e graxas extraídos visando a conversão em biocombustíveis. Química Nova, 37, 597 - 602.

OLIVEIRA, S. C., BARROS, S. T. D., GIMENES, M. L. 2013. The extraction of passion fruit oil with green solvents. Journal of Food Engineering, 117, 458 - 463.

RAMLUCKAN, K., MOODLEY, K.G., BUX, F. 2014. An evaluation of the efficacy of using selected solvents for the extraction of lipids from algal biomass by the soxhlet extraction method.

Fuel, 116, 103-108.

REZAIE, M., FARHOOSH, R., IRANSHAHI, M., SHARIF, A., GOLMOHAMADZADEH, S.

2015. Ultrasonic-assisted extraction of antioxidative compounds from Bene (Pistacia atlantica subsp. mutica) hull using various solvents of different physicochemical properties. Food Chemistry, 173, 577-583. 
ROSCOE, R., RICHETTI, A., MARANHO, E. 2007. Análise de viabilidade técnica de oleaginosas para produção de biodiesel em Mato Grosso do Sul. Revista de Política Agrícola, 16, 48-59. SAMARAM, S., MERHSSEINI, H., TAN, C. P., GHAZALI, H. M., BORDBAR, S., SERJOUUIE. 2015. Optimization of ultrasound-assisted extraction of oil from papaya seed by response surface methodology: Oil recovery, radical scavenging antioxidant activity, and oxidation stability. Food Chemistry, 172, 7-17.

SOUZA, A. D. V., FÁVARO, S. P., ÍTAVO, L. C. V., ROSCOE, R. 2009. Caracterização química de sementes e tortas de pinhão-manso, nabo-forrageiro e crambe. Pesquisa Agropecuária Brasileira, 44, 1328-1335.

SUGANYA, T., RENGANATHAN, S. 2012. Optimization and kinetic studies on algal oil extraction from marine macroalgae Ulva lactuca. Bioresource Technology, 107, 319-326.

STANISAVLJEVIĆ, I. T., LAZIC, M. L., VELJKOVIC. 2007. Ultrasonic extraction of oil from tobacco (Nicotiana tabacum L.) seeds. Ultrasonics Sonochemistry, 14, 646 - 652.

TIAN, Y., XU, Z., ZHENG, B., LO, Y. M. 2013. Optimization of ultrasonic-assisted extraction of pomegranate (Punicagranatum L.) seed oil. Ultrasonics Sonochemistry, 20, 202-208.

ULUATA, S., OZDEMIR, N. 2012. Antioxidant Activities and Oxidative Stabilities of Some Unconventional Oil seeds. Journal of the American Oil Chemists' Society, 89, 551-559.

VALLE, P., VELEZ, A., HEGEL, P., MABE, G., BRIGNOLE, E. A. 2010. Biodiesel production using supercritical alcohols with a non-edible vegetable oil in a batch reactor. The Journal of Supercritical Fluids, 54, 61-70.

ZHANG, Y. S., ZHAO, Y. W. 2006. Study on ultrasonic wave Extraction of kiwifruit seed oil. Journal of the Chinese Cereals and Oils Association, 21, 116-118.

ZHANG, Q. A., ZHAO, Z. Q. YUE, X. F., FAN, X. H., LI, T., CHEN, S. F. 2009. Response surface optimization of ultrasound-assisted oil extraction from autoclaved almond powder. Food Chemistry, 116, 513-518.

WANG, Y. P., TANG, J. S., CHU, C. Q., TIAN, J. 2000. A preliminary study on the introduction and cultivation of Crambe abyssinica in China, an oil plant for industrial uses. Industrial Crops and Products, 12, 47-52. 